\title{
Efficacy of pethidine, ketorolac, and lidocaine gel as analgesics for pain control in shockwave lithotripsy: A single-blinded randomized controlled trial
}

\author{
Abdelwahab Hashem, Fady K. Ghobrial, M. A. Elbaset, Ahmed M. Atwa, Mohamed Fadallah, Mahmoud Laymon, \\ Ahmed El-Assmy, Khaled Z. Sheir, Hassan Abol-Enein \\ Department of Urology, Urology and Nephrology Center, Mansoura University, Mansoura, Egypt
}

Purpose: To compare the safety and efficacy of xylocaine gel and ketorolac as opioid-sparing analgesia compared with pethidine for shock wave lithotripsy (SWL) pain.

Materials and Methods: A single-blinded randomized controlled trial (RCT) was performed in 132 patients with renal and upper ureteral stones amenable to treatment with SWL. The first patient group received intravenous (IV) pethidine and placebo gel; the second group received IV ketorolac plus placebo gel; the third group received lidocaine gel locally plus normal saline IV. Stone disintegration was classified as none (no change from basal by kidney, ureter, bladder X-ray or ultrasound [US] imaging), partial (fragmented and $>4$-mm residual fragments), and complete ( $\leq 4-\mathrm{mm}$ residual fragments). Stone disintegration was assessed by kidneyureter-bladder X-ray and US imaging. Pain was evaluated by use of the Numeric Pain Rating Scale (NPRS).

Results: The NPRS scores were highest in the xylocaine group at 10, 20, and 30 minutes ( $p=0.0001$ ) with no significant difference between the ketorolac and pethidine groups, except at 10 minutes $(p=0.03)$ and a near significant difference at 30 minutes $(p=0.054)$ in favor of ketorolac. Results for stone disintegration (none, partial, and complete, respectively) were as follows: 25 $(50.0 \%), 23(46.0 \%)$, and $2(4.0 \%)$ for pethidine; $19(35.8 \%), 23(43.4 \%)$, and $11(20.8 \%)$ for ketorolac; and $26(89.7 \%), 3(10.3 \%)$, and $0(0.0 \%)$ for lidocaine $(\mathrm{p}=0.008)$.

Conclusions: Ketorolac is a safe and more effective alternative to morphine derivatives for SWL analgesia. Lidocaine gel should not be used as mono-analgesia for SWL.

Keywords: Analgesia; Ketorolac; Lithotripsy

This is an Open Access article distributed under the terms of the Creative Commons Attribution Non-Commercial License (http://creativecommons.org/licenses/by-nc/4.0) which permits unrestricted non-commercial use, distribution, and reproduction in any medium, provided the original work is properly cited.

Received: 25 December, 2018 - Accepted: 7 April, 2019

Corresponding Author: Abdelwahab Hashem

Department of Urology, Urology and Nephrology Center, Mansoura University, El Gomhoureya St., El Mansoura 35516, Egypt

TEL: +20-1069678979, FAX: +20-50-2263717, E-mail: abdelwahab_hashem@yahoo.com

ORCID: https://orcid.org/0000-0002-0664-3504 


\section{INTRODUCTION}

The management of urolithiasis has been revolutionized with the introduction of shock wave lithotripsy (SWL) owing to its simplicity, noninvasive nature, efficacy, and minimal morbidity. Pain relief during SWL is vital, not only to maintain patient comfort and satisfaction, but also to facilitate stone imaging and targeting by reducing patient movement during successive shock wave impacts. Reduced patient movement increases the efficiency of fragmentation and reduces morbidity from SWL. The perception of pain during SWL is affected by patient-related factors like age, sex, and body habitus. Also, young female patients, anxious and depressed patients, and thin patients experience more pain during SWL [1-3].

Analgesia for SWL includes intravenous (IV) propofol, extradural and epidural anesthetics, nitrous oxide, local anesthetic injection, and EMLA creams (a eutectic mixture of local anesthetics). Analgesics including paracetamol, nonsteroidal anti-inflammatory drugs (NSAIDs), and opioids have also been found to provide sufficient pain control during SWL. Nontraditional methods such as music, acupuncture, and transcutaneous electrical nerve stimulation have also been used [4].

Although European guidelines state that pain control during SWL is necessary, no recommendation is made for a specified analgesia, in contrast with a clear recommendation for pain relief for an acute renal pain episode [5]. Consequently, 17 distinct analgesia regimens were used in 21 centers for SWL analgesia in the United Kingdom. More than one regimen was used in $50 \%$ of these centers. Diclofenac followed by pethidine and paracetamol were the most frequently used. IV fentanyl patient-controlled analgesia was used in $10.0 \%$ of the centers [6]

In our SWL unit, pethidine is the standard analgesic used. We aimed to compare it with other opioid-sparing techniques, specifically, xylocaine gel and ketorolac, a potent analgesic with moderate anti-inflammatory activity with greater efficacy than that of morphine and pethidine [7].

\section{MATERIALS AND METHODS}

This was a single-blinded randomized controlled trial (RCT) and was registered at NIH ClinicalTrials.gov (http:// www.clinicaltrials.gov/; NCT03032458). Patients were aged $\geq 18$ years with renal and upper ureteral stones amenable to SWL according to guidelines [5] at the SWL unit at the Urology and Nephrology Center between January and October 2017. Allergy to pethidine, ketorolac, or xylocaine gel; an American Society of Anesthesiologists score $\geq 4$; and body mass index $\geq 40 \mathrm{~kg} / \mathrm{m}^{2}$ were added as exclusion criteria [5]. The CONSORT flowchart is shown in Fig. 1.

Patients were divided into three groups by use of computer-generated sequentially numbered randomization: the first group received pethidine (pethidine $25 \mathrm{mg} / \mathrm{mL}$ ampule (amp.) British Pharmacopoeia 2002; Misr, Cairo, Egypt) as an IV bolus injection before the session plus a placebo gel and then an IV infusion, so that the total dose did not exceed $1 \mathrm{mg} / \mathrm{kg}$. The second group received an IV bolus injection of ketorolac (ketorolac $30 \mathrm{mg} / 2 \mathrm{~mL}$ amp., Amriya, Alexandria, Egypt) before the session plus a placebo gel and then a 30-mg IV infusion so that the total dose did not exceed $60 \mathrm{mg}$. The third group received a xylocaine gel (2\% lidocaine gel, Astra Zeneca, Södertälje, Sweden) locally before the session with a 10-mg saline IV bolus before the session and then an IV saline infusion after. The drug doses were based on previous reviews and meta-analysis for postoperative pain control doses [8,9]. The patients were blinded as to the used drug. An adjuvant anti-emetic was used if needed.

After the routine pre-procedural evaluation, SWL was performed using a Dornier lithotripter S (Dornier MedTech, Weßling, Germany) started at a power of $12 \mathrm{kV}$ and then gradually increased to $17 \mathrm{kV}$. A total of 3,000 shocks was delivered per session or until complete fragmentation of the stone. The study primary outcome was to compare the efficacy of pethidine, ketorolac, and xylocaine gel in SWL analgesia. A validated 0 to 10 Numeric Pain Rating Scale (NPRS) [10] was used for pain evaluation. The secondary outcome was to assess stone disintegration 2 weeks after the SWL session, SWL complications, and SWL session patientreported satisfaction between the groups. A single 4-tiered question regarding patient overall self-reported satisfaction about the efficacy of the analgesia efficacy was used (very satisfied, satisfied, unsatisfied, and very unsatisfied). Measurements of vital parameters during the SWL session and complications were reported. A kidney-ureter-bladder (KUB) X-ray and ultrasound (US) scan (2 weeks after a single SWL session) were used to assess stone disintegration. Noncontrast computed tomography (NCCT) was done for patients with no residual detected by KUB and US. Stone disintegration was classified as none (no change from basal by KUB X-ray or US imaging), partial (fragmented and $>4$-mm residual fragments), and complete ( $\leq 4-\mathrm{mm}$ residual fragments).

The power of the study was calculated by using the G*Power program (University of Düsseldorf, Düsseldorf, Germany) to determine an adequate sample size based on 
previous trials [11,12]. Using the priori test with accuracy mode calculation and an effect size convention of 0.35 for the one-way ANOVA, with a error probability of 0.05 , provided $95 \%$ power with expected $20 \%$ dropout rate for a total sample size of 165 patients. Statistical analysis was performed with the use of the IBM SPSS Statistics ver. 22.0 for Windows (IBM Corp., Armonk, NY, USA). Statistical analyses were done by using one-way ANOVA, and chi-square tests, and use of Bonferroni post-hoc tests to determine the difference between the two groups. A p-value $<0.05$ was considered to be statistically significant.

All procedures in this study involving human participants were in accordance with the ethical standards of the Mansoura Faculty of Medicine Institutional Research Board (approval number: MD/161172) and with the 1964 Helsinki declaration and its later amendments or comparable ethical standards. Informed consent was obtained from all participants included in the study.

\section{RESULTS}

Patient data and stone characteristics of the three groups as shown in Table 1 did not differ significantly. In the xylocaine group, six sessions (20.7\%) were aborted and a supplemental analgesic (our standard regimen was pethidine) was added. Also, most patients in the xylocaine group showed the least satisfaction with pain control; therefore, we stopped allocating patients to this arm. All monitored vital parameters of the patients were comparable between groups.

Parameters of the SWL sessions with pain ratings are shown in Table 2. The NPRS scores were significantly different between groups. By use of the past-hoc test, NPRS scores were highest in the xylocaine group at 10,20, and 30 minutes $(p=0.0001)$. However, there was no significant difference between the ketorolac and pethidine groups, except at 10 minutes $(p=0.03)$ and a near significant difference at 30 minutes $(p=0.054)$ in favor of ketorolac. In view of the SWL parameters, the number of shocks and

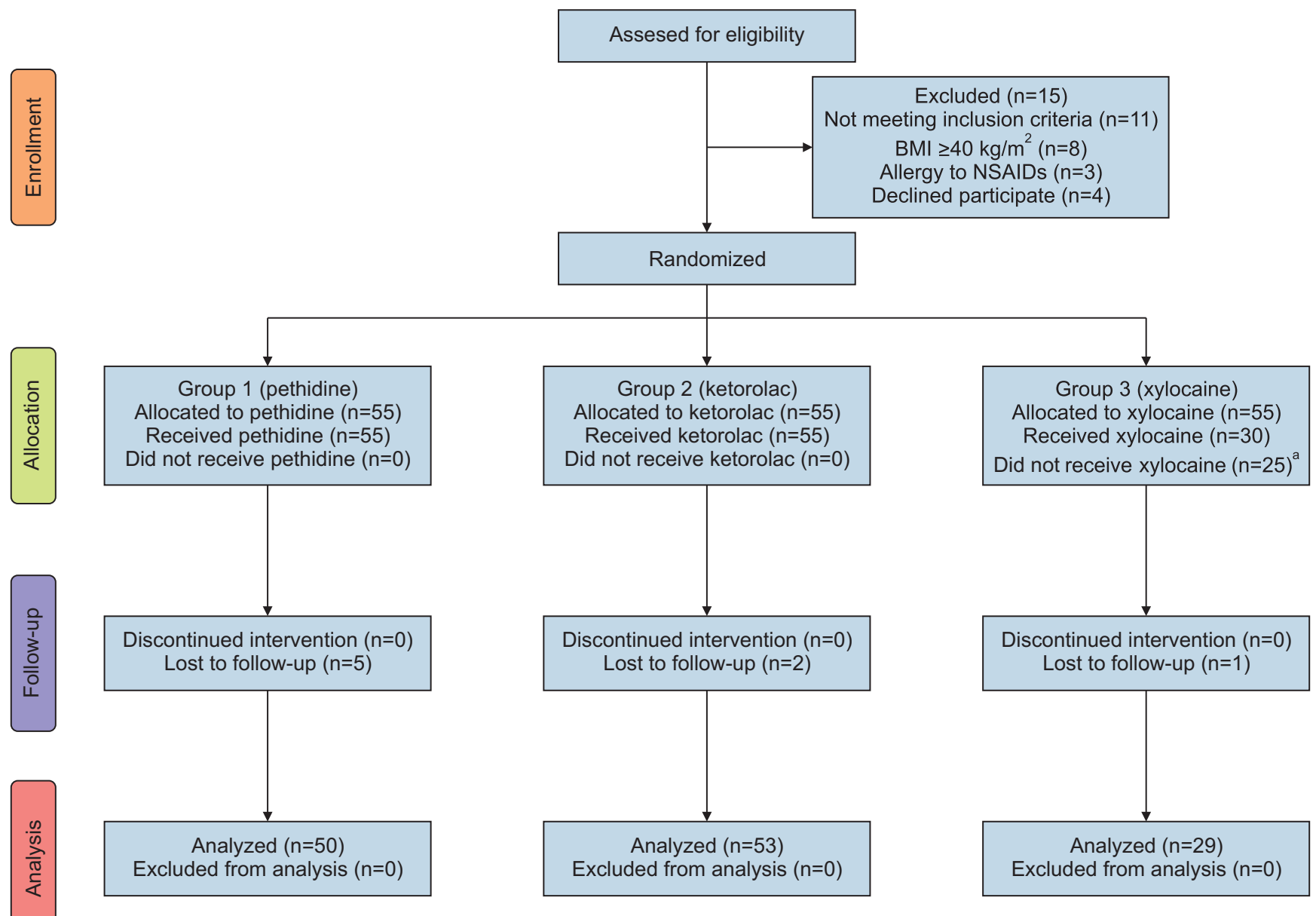

Fig. 1. CONSORT flowchart for study participants. BMI, body mass index; NSAIDs, nonsteroidal anti-inflammatory drugs. ${ }^{\text {a }}$ :These patients received pethidine (our standard analgesia) and were excluded from our final analysis. 
Table 1. Patient demographic characteristics and stone characteristics in the three groups

\begin{tabular}{|c|c|c|c|c|}
\hline Variable & Pethidine $(n=50)$ & Ketorolac $(n=53)$ & Xylocaine gel $(n=29)$ & $p$-value \\
\hline Age (y) & $45.2 \pm 12.9$ & $45.9 \pm 12.5$ & $46.9 \pm 11.7$ & $0.8^{\mathrm{a}}$ \\
\hline Sex & & & & $0.4^{\mathrm{b}}$ \\
\hline Male & 24 & 28 & 13 & \\
\hline Female & 26 & 25 & 16 & \\
\hline $\mathrm{BMI}\left(\mathrm{kg} / \mathrm{m}^{2}\right)$ & $29.1 \pm 4.8$ & $29.4 \pm 4.8$ & $30.2 \pm 4.9$ & $0.2^{\mathrm{a}}$ \\
\hline ASA score & & & & $0.5^{\mathrm{b}}$ \\
\hline I & $43(86.0)$ & $40(75.5)$ & $23(79.3)$ & \\
\hline II & $7(14.0)$ & $11(20.8)$ & $5(17.2)$ & \\
\hline III & $0(0.0)$ & $2(3.8)$ & $1(3.4)$ & \\
\hline Diabetes mellitus & $2(4.0)$ & $3(5.7)$ & $3(10.3)$ & $0.5^{\mathrm{b}}$ \\
\hline Serum creatinine $(\mathrm{mg} / \mathrm{dL})$ & $0.9 \pm 0.3$ & $0.9 \pm 0.2$ & $0.9 \pm 0.2$ & $0.9^{\mathrm{a}}$ \\
\hline Stone & & & & $0.3^{\mathrm{b}}$ \\
\hline Single & $34(68.0)$ & $42(79.2)$ & $24(82.8)$ & \\
\hline Multiple & $16(32.0)$ & $11(20.8)$ & $5(17.2)$ & \\
\hline Stone opacity & & & & $0.9^{b}$ \\
\hline Opaque & $46(92.0)$ & $49(92.5)$ & $27(93.1)$ & \\
\hline Lucent & $4(8.0)$ & $4(7.5)$ & $2(6.9)$ & \\
\hline Stone volume $\left(\mathrm{mm}^{3}\right)$ & $0.5(0.08-5.1)$ & $0.45(0.06-4.3)$ & $0.33(0.09-1.12)$ & $0.1^{\mathrm{a}}$ \\
\hline Stone to skin distance $(\mathrm{cm})$ & $10.2 \pm 2.5$ & $10.5 \pm 2.4$ & $10.8 \pm 2.6$ & $0.5^{\mathrm{a}}$ \\
\hline Renal cortical thickness $(\mathrm{cm})$ & $1.9 \pm 0.6$ & $1.9 \pm 0.5$ & $1.9 \pm 0.4$ & $0.8^{\mathrm{a}}$ \\
\hline Muscle thickness (cm) & $5.7 \pm 2$ & $6 \pm 2$ & $5.7 \pm 2.3$ & $0.7^{\mathrm{a}}$ \\
\hline Soft tissue thickness (cm) & $7.5 \pm 2.5$ & $7.6 \pm 2.4$ & $8.2 \pm 2.9$ & $0.4^{\mathrm{a}}$ \\
\hline Average hounsfield units & $739.3 \pm 269.5$ & $769 \pm 280.2$ & $711 \pm 251.2$ & $0.6^{\mathrm{a}}$ \\
\hline
\end{tabular}

Values are presented as mean \pm standard deviation, number only, number (\%), or median (range).

BMI, body mass index; ASA, American Society of Anesthesiologists.

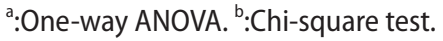

Table 2. Characteristics of the shock wave sessions and patients' reactions

\begin{tabular}{|c|c|c|c|c|}
\hline Characteristic & Pethidine $(n=50)$ & Ketorolac $(n=53)$ & Xylocaine gel $(n=29)$ & p-value \\
\hline Power (kJ) & $6.1 \pm 1.3$ & $6.5 \pm 1.1$ & $5.3 \pm 1.6$ & $0.0001^{\circ}$ \\
\hline Total energy (watt) & $98.2 \pm 26.5$ & $111.8 \pm 23.2$ & $86.3 \pm 32.5$ & $0.0001^{\circ}$ \\
\hline No of shocks & $2,936 \pm 226.6$ & $2,981.1 \pm 139.4$ & $2,027.6 \pm 908.3$ & $0.0001^{\circ}$ \\
\hline Session time (min) & $30 \pm 0$ & $31 \pm 3.0$ & $22.9 \pm 7.3$ & $0.0001^{\circ}$ \\
\hline NPRS-10 & $1.88 \pm 0.59$ & $0.96 \pm 0.47$ & $3.79 \pm 1.62$ & $0.0001^{\circ}$ \\
\hline NPRS-20 & $2.62 \pm 1.18$ & $1.96 \pm 0.85$ & $5.42 \pm 2.04$ & $0.0001^{\circ}$ \\
\hline NPRS-30 & $2.98 \pm 1.32$ & $1.83 \pm 0.64$ & $6.20 \pm 2.69$ & $0.0001^{\circ}$ \\
\hline Supplementary analgesia (add-on) & $0(0.0)$ & $0(0.0)$ & $6(20.7)$ & $0.0001^{b}$ \\
\hline Nausea, vomiting & $18(36.0)$ & $0(0.0)$ & $2(6.9)$ & $0.0001^{b}$ \\
\hline Post SWL complications & & & & $0.3^{\mathrm{b}}$ \\
\hline Hematuria & 2 & 3 & 1 & \\
\hline Steinstrasse & 0 & 1 & 0 & \\
\hline Perinephric hematoma & 1 & 1 & 1 & \\
\hline \multicolumn{5}{|l|}{ Satisfaction } \\
\hline Yes (very satisfied, satisfied) & $37(74.0)$ & $46(86.8)$ & $5(17.2)$ & $0.0001^{b}$ \\
\hline
\end{tabular}

Values are presented as mean \pm standard deviation, number (\%), or number only. NPRS, Numeric Pain Rating Scale; SWL, shock wave lithotripsy.

a :One-way ANOVA. ${ }^{\mathrm{b}}$ :Chi-square test. 
total energy showed some significant differences. By use of the post-hoc test, patients in the xylocaine group had the least ability to tolerate shock numbers and total energy $(p=0.0001)$, with no significant difference between the ketorolac and pethidine groups $(\mathrm{p}=1.0)$ in shocks numbers; however, patients in the ketorolac group tolerated more energy than did patients in the pethidine group $(\mathrm{p}=0.04)$.

In our RCT, we considered the patients satisfied if their answers were "very satisfied" or "satisfied." Patient selfreports of satisfaction did not differ significantly between the ketorolac and pethidine groups $(\mathrm{p}=0.13)$. Xylocaine had the lowest satisfaction values among the groups $(p=0.0001$, Table 2). A total of $36.0 \%$ of patients in the pethidine group experienced nausea and/or vomiting compared with only $6.9 \%$ of patients in the xylocaine group and none in the ketorolac group ( $p<0.0001)$.

Post-SWL complications did not reveal any significant differences in renal hematoma, a drop in hemoglobin, or consequently blood transfusion between the groups $(\mathrm{p}=0.3$ ). We did not assess the stone-free rate in this RCT, because NCCT was used only in patients who showed complete disintegration (13, 9.8\%), whereas US and KUB were done for patients with none and partial disintegration. Also, our policy is to assess the stone-free rate after the last session (maximum $\mathrm{n}=4$ ) with an interval of 2 weeks in between.

Regarding stone disintegration, the rate was lowest in the xylocaine group, with 26 patients with none (89.7\%), 3 with partial (10.3\%), and 0 with complete $(0.0 \%)$ disintegration of the stone $(\mathrm{p}=0.008)$. The rate of stone disintegration was better in the ketorolac group than in the pethidine group $(\mathrm{p}=0.031)$.

\section{DISCUSSION}

SWL has revolutionized the treatment of nephrolithiasis worldwide. SWL is a noninvasive or extremely low-invasive treatment that, in most patients, can be carried out without general or regional anesthesia on an outpatient basis [13]. The success of SWL depends on the efficacy of the lithotripter and the following factors: size, location, and composition of the stones; patient's habitus; and performance of SWL [5]. Pain control is one of these factors and significantly influences retreatment rate and final outcome of SWL. European guidelines state that careful control of pain during treatment is necessary to limit pain-induced movements and excessive respiratory excursions [5].

To date, the pathogenesis of SWL pain is not clear. Stimulation of the superficial nociceptors in the skin as well as the deeper, visceral nociceptors in the renal capsule, periosteum, pleura, peritoneum, and muscles caused by the shock wave-generated microbubbles in body fluids play a role in the SWL pain pathway. Moreover, stone movement during the session can increase pain [14]

Different analgesia regimens have been reported in SWL analgesia practice. Therefore, it is crucial to choose an adequate analgesic with few adverse effects. Sorensen et al. [15] reported that the 3-month stone-free rate for general anesthesia was $87 \%$ compared with a stone-free rate of $55 \%$ if IV sedation was used for SWL analgesia $(\mathrm{p}<0.001)$. On the contrary, about one third of urologic patients had cardiopulmonary problems, including medically compromised patients or elderly patients that limit the use of standard anesthetic techniques. Sedoanalgesia was developed in urologic surgery in response to the increasing demand for cost-effective, minimally invasive surgery, office surgery, and rapid complication-free recovery [16].

Pethidine is a synthetic opioid that exerts its analgesic effects by acting as an agonist at $\mu$-receptors. In a doubleblinded RCT, morphine and pethidine provided effective postoperative analgesia with an acceptable adverse effect profile, whereas tramadol had acceptable analgesia but required more rescue analgesic doses [17]. Although pethidine showed at least a $50 \%$ decrease in acute postoperative pain, $68 \%$ of patients experienced adverse effects like drowsiness, dizziness, nausea, or vomiting as reported in a systematic review with meta-analysis [8]. In the current study, the pethidine group had a mean NPRS score of 2.5 regarding pain control. About $36 \%$ of patients in the pethidine group experienced adverse effects. The stone disintegration results with pethidine usage were as follows: none, 25 (50.0\%); partial, 23 (46.0\%); and complete, 2 (4.0\%). About three quarters of the patients were satisfied.

Opioid-sparing techniques have been recognized as an important component of postoperative pain management. Ketorolac is a NSAID with analgesic properties. A 60$\mathrm{mg}$ intramuscular injection of ketorolac has a longer time to peak concentration (30-50 minutes) than does a 30 mg IV dose (3-5 minutes). The 60 -mg dose reduces early postoperative pain, has opioid-sparing effects, and reduces postoperative nausea and vomiting, with lack of evidence of these effects for the 30-mg dose. Regarding safety, ketorolac does not show an increase in abnormal bleeding, blood transfusions, or gastritis symptoms [9] In our RCT, ketorolac did not show a significant increase in renal hematoma, a drop in hemoglobin, or consequent blood transfusion.

In a systematic review with meta-analysis by Smith et al. [8], for single-dose ketorolac and pethidine in acute postoperative pain, the authors found that the number- 
needed-to-treat (NNT) for pethidine $100 \mathrm{mg}$ was 2.9 (95\% confidence interval [CI], 3.2-3.9), and 53.6\% had at least 50\% pain relief. The NNT for 30-mg ketorolac was 3.4 (95\% CI, $2.5-4.9$ ), and $52.8 \%$ of patients had at least $50 \%$ pain relief. However, with the 60-mg ketorolac dose, the NNT was 1.8 (95\% CI, $15-2.3)$, and $55.5 \%$ of patients had at least $50 \%$ pain relief.

In our series, the ketorolac group had a tolerable pain rating with no reported adverse effects during SWL sessions. Ketorolac had favorable stone disintegration results: none, 19 (35.8\%); partial, 23 (43.4\%); and complete, 11 (20.8\%), respectively $(\mathrm{p}=0.008)$. Patient satisfaction was more than 85\%. Akcali et al. [18] concluded that lornoxicam, an NSAID, can be safely and efficiently preferred in pain control during SWL. In a recent meta-analysis that assessed analgesia for patients undergoing SWL, no significant difference in pain scores was reported between NSAIDs or opioids [4]

EMLA cream containing lidocaine and prilocaine is a topical anesthetic drug designed for use on intact skin. EMLA cream has been used for SWL sedoanalgesia with conflicting results [12,19]. In one RCT, EMLA cream was reported to be as safe and effective as fentanyl, diclofenac, and tramadol with no statistically significant differences regarding pain scores or adverse effects [12]. However, in the other RCT, EMLA cream did not significantly modify pain perceived and did not have any significant opioid-sparing effect [19]. In our study, we used lidocaine instead of EMLA cream owing to its availability in our center.

Lidocaine requires an injection, a painful process, to conduct deep anesthesia. Transdermal drug delivery, known as sonophoresis, is a noninvasive alternative to local injection by use of US [20]. Luh et al. [21] proved that pretreatment extracorporeal shock wave therapy (ESWT) and concurrent ESWT accelerate the anesthetic effects of local anesthetic In this study, xylocaine (lidocaine) gel had the highest NPRS scores. A total of $20 \%$ of patients stopped the SWL session due to pain. Patient satisfaction was only $17 \%$ in the xylocaine group. This higher rate of pain associated with patient movement, beside the aborted SWL in 6 sessions (20.7\%), might have caused the unfavorable stone disintegration results in the xylocaine group, which were as follows: none, 26 (89.7\%); partial, 3 (10.3\%); and complete, 0 $(0.0 \%)$, respectively $(\mathrm{p}=0.008)$.

Although our study was an RCT, it had some limitations. Not all patients underwent NCCT for assessment of the stone-free rate. Also, we tested these drugs during a single SWL session in a small number of patients. Patientreported satisfaction was not assessed by use of a validated questionnaire. Additional larger trials are needed to assess a combination of multiple regimens of analgesia in a large number of patients.

\section{CONCLUSIONS}

Ketorolac is safe and a more effective alternative to morphine derivatives for SWL analgesia. Lidocaine gel should not be used as mono-analgesia for SWL.

\section{CONFLICTS OF INTEREST}

The authors have nothing to disclose.

\section{REFERENCES}

1. Salinas AS, Lorenzo-Romero J, Segura M, Calero MR, Hernández-Millán I, Martínez-Martín M, et al. Factors determining analgesic and sedative drug requirements during extracorporeal shock wave lithotripsy. Urol Int 1999;63:92-101.

2. Vergnolles M, Wallerand H, Gadrat F, Maurice-Tison S, Deti E, Ballanger $\mathrm{P}$, et al. Predictive risk factors for pain during extracorporeal shockwave lithotripsy. J Endourol 2009;23:2021-7.

3. Berwin JT, El-Husseiny T, Papatsoris AG, Hajdinjak T, Masood J, Buchholz N. Pain in extracorporeal shock wave lithotripsy. Urol Res 2009;37:51-3.

4. Aboumarzouk OM, Hasan R, Tasleem A, Mariappan M, Hutton R, Fitzpatrick J, et al. Analgesia for patients undergoing shockwave lithotripsy for urinary stones-a systematic review and meta-analysis. Int Braz J Urol 2017;43:394-406.

5. European Association of Urology. The EAU urolithiasis guidelines [Internet]. Arnhem: European Association of Urology; 2018 Jun 18 [cited 2018 Jun 23]. Available from: http://uroweb. org/guideline/urolithiasis/.

6. Sharma NL, Alexander CE, Grout E, Turney BW. Shockwave lithotripsy: variance within UK practice. Urolithiasis 2017;45:193-201.

7. Buckley MM, Brogden RN. Ketorolac. A review of its pharmacodynamic and pharmacokinetic properties, and therapeutic potential. Drugs 1990;39:86-109.

8. Smith LA, Carroll D, Edwards JE, Moore RA, McQuay HJ. Single-dose ketorolac and pethidine in acute postoperative pain: systematic review with meta-analysis. Br J Anaesth 2000;84:4858.

9. De Oliveira GS Jr, Agarwal D, Benzon HT. Perioperative single dose ketorolac to prevent postoperative pain: a meta-analysis of randomized trials. Anesth Analg 2012;114:424-33.

10. Castarlenas E, Jensen MP, von Baeyer CL, Miró J. Psychometric properties of the numerical rating scale to assess self-reported pain intensity in children and adolescents: a systematic review. 
Clin J Pain 2017;33:376-83.

11. Takmaz SA, Inan N, Goktug A, Erdogan I, Sunay M, Ceyhan A. The analgesic effect of 8 and $16 \mathrm{mg}$ lornoxicam administered before shock wave lithotripsy: a randomized, double-blind, controlled study. Urology 2008;72:282-5.

12. Basar H, Yilmaz E, Ozcan S, Buyukkocak U, Sari F, Apan A, et al. Four analgesic techniques for shockwave lithotripsy: eutectic mixture local anesthetic is a good alternative. J Endourol 2003;17:3-6.

13. Christian C, Thorsten B. The preferred treatment for upper tract stones is extracorporeal shock wave lithotripsy (ESWL) or ureteroscopic: pro ESWL. Urology 2009;74:259-62.

14. Bach C, Zaman F, Kachrilas S, Kumar P, Buchholz N, Masood J. Drugs for pain management in shock wave lithotripsy. Pain Res Treat 2011;2011:259426.

15. Sorensen C, Chandhoke P, Moore M, Wolf C, Sarram A. Comparison of intravenous sedation versus general anesthesia on the efficacy of the Doli 50 lithotriptor. J Urol 2002;168:35-7.

16. Birch BR, Anson KM, Miller RA. Sedoanalgesia in urology: a safe, cost-effective alternative to general anaesthesia. a review of 1020 cases. Br J Urol 1990;66:342-50.
17. Unlugenc $H$, Vardar MA, Tetiker S. A comparative study of the analgesic effect of patient-controlled morphine, pethidine, and tramadol for postoperative pain management after abdominal hysterectomy. Anesth Analg 2008;106:309-12.

18. Akcali GE, Iskender A, Demiraran Y, Kayikci A, Yalcin GS, Cam K, et al. Randomized comparison of efficacy of paracetamol, lornoxicam, and tramadol representing three different groups of analgesics for pain control in extracorporeal shockwave lithotripsy. J Endourol 2010;24:615-20.

19. Ganapathy S, Razvi H, Moote C, Parkin J, Yee I, Gverzdys $S$, et al. Eutectic mixture of local anaesthetics is not effective for extracorporeal shock wave lithotripsy. Can J Anaesth 1996;43:1030-4.

20. Escobar-Chávez JJ, Bonilla-Martínez D, Villegas-González MA, Rodríguez-Cruz IM, Domínguez-Delgado CL. The use of sonophoresis in the administration of drugs throughout the skin. J Pharm Pharm Sci 2009;12:88-115.

21. Luh JJ, Huang WT, Lin KH, Huang YY, Kuo PL, Chen WS. Effects of extracorporeal shock wave-mediated transdermal local anesthetic drug delivery on rat caudal nerves. Ultrasound Med Biol 2018;44:214-22. 